\title{
ESTUDIO COMPARATIVO ENTRE EL CAPITAL INTELECTUAL DE TRABAJADORES DE LAS GENERACIONES $X$ y $Y$ EN EL ÁREA METROPOLITANA DE BUCARAMANGA, COLOMBIA.
}

\section{Comparative study between the intellectual capital of workers of generations $\mathrm{X}$ and $\mathrm{Y}$ in the metropolitan area of Bucaramanga, Colombia.}

\section{Enghi Zoraya Medellín-Galvis}

Estudiante de Ingeniería Industrial de la Universidad de Investigación y Desarrollo en la ciudad de Bucaramanga

(Colombia). Correo electrónico: emedellin1@udi.edu.co

\section{Julián David Molina-Hernández \\ Estudiante de Ingeniería Industrial de la Universidad de Investigación y Desarrollo en la ciudad de Bucaramanga} (Colombia). Correo electrónico: imolina2@udi.edu.co

\section{Sebastián García-Méndez}

Docente, Magíster en Administración, profesor de la Universidad de Investigación y Desarrollo en la ciudad de Bucaramanga (Colombia). Correo electrónico: sgarcia15@udi.edu.co

| Recibido/Received: 13/01/2021 • Aprobado/Approved: 29/05/2021 • Publicado/Published: 20/06/2021|

\section{Resumen}

La presente investigación tiene como objeto presentar una comparación que tenga como base el capital intelectual, en una muestra de colaboradores de Bucaramanga y de su área metropolitana, considerando como criterio de inclusión la pertenencia a las generaciones X y Y. Para tal fin, se aplicó un cuestionario basado en el modelo intelect, considerando las dimensiones de capital humano, capital relacional y capital estructural; posteriormente se desarrolló la prueba $U$ de Mann-Withney. Los resultados obtenidos permiten determinar que no existe una diferencia significativa en las dimensiones objeto de estudio, lo cual posibilita generalizar diversas prácticas en las organizaciones, dentro del marco que permite lograr la competitividad y productividad deseada en los mercados específicos.

Palabras clave: capital intelectual; generación X; generación Y; trabajadores; Bucaramanga.

\section{Abstract}

The purpose of this research is to present a comparison based on intellectual capital in a sample of collaborators from Bucaramanga and its metropolitan area, considering as inclusion criteria the belonging to generations $\mathrm{X}$ and $\mathrm{Y}$. For this purpose, a questionnaire based on the intelect model was applied, considering the dimensions of human capital, relational capital and structural capital; subsequently, the Mann-Withney $U$ test was developed. The results obtained show that there is no significant difference in the dimensions under study, which makes it possible to generalize 
different practices in organizations, within the framework that allows them to achieve the desired competitiveness and productivity in specific markets.

Key words: intellectual capital; generation X; generation Y; workers; Bucaramanga.

\section{Introducción}

La Real Academia Española (RAE, s. f.) define el término "generación", como un grupo de individuos que comparten acontecimientos históricos, comportamientos y creencias, y que además han vivido la misma época, por lo cual mantienen un sentimiento de pertenencia, el cual permite una diferenciación de ese grupo en particular, con los demás grupos generacionales. Existen distintas generaciones, pero en la actualidad, solo existen cuatro grupos generacionales que se encuentran laborando en las organizaciones. Oblinger \& Lippincott (2005), mencionan que dentro de la fuerza laboral actual se encuentran los baby boomers (nacidos entre 1946-1964); la generación X (nacidos entre 1965-1980); la generación Y (también conocida como los millennnials, nacidos entre 19811994) y la generación $Z$ (nacidos a partir de 1995 hasta la actualidad). En vista de que los baby boomers están próximos a concluir su etapa laboral debido a su edad, y que la generación $Z$ corresponde a jóvenes que en su mayoría están en su etapa de formación profesional e iniciando su etapa laboral, este estudio se enfocará en la generación $X$ y en la generación $Y$, dado que las mismas se encuentran bien establecidas dentro de las organizaciones, y que ambas son las que deben trabajar en conjunto, en pro del cumplimiento de los objetivos empresariales, mediados por factores del contexto (García-Méndez et al., 2020). A su vez, se ha de tener en cuenta que el choque generacional es un factor crítico que dificulta las relaciones laborales. Particularmente en Colombia, los millennials ocupan alrededor del $60 \%$ de los cargos, por lo que se presenta una dificultad al interactuar, al convivir, al compartir, al trabajar en equipo y al tomar decisiones consensuadas con las demás generaciones; por ello, el desafío se encuentra en hacer del aprovechamiento de las diferencias, el verdadero capital de las organizaciones(Sarkis, 2017).

En ese mismo sentido, cada generación es fiel a sus principios y a su forma de pensar; por lo tanto, es necesario tener el conocimiento adecuado sobre su capital intelectual, pues según Azofra et al., 2017, lo anterior se constituye como un habilitador relevante en el aprendizaje, en la motivación, en la generación de recursos intangibles y en la creación de valor. Así mismo, segúnLimache (2017) el capital intelectual influye en la competitividad de las organizaciones, en tanto favorece factores como la mejoría de su posicionamiento frente a la competencia; el logro de tener mayores utilidades y la capacidad de poseer menor endeudamiento así como la favorabilidad de utilizar de manera óptima la capacidad de la planta y de tener menor rotación de los individuos. Es resumidas cuentas, permite, maximizar los recursos disponibles de la organización, traduciéndose ello en lograr mayores beneficios corporativos (Gómez, Aristizábal \& Fuentes, 2017).

De acuerdo con lo anterior, es en ese contexto en el cual se desarrolla la investigación, la cual se 
encuentra referida al análisis del capital intelectual de las generaciones $X$ y $Y$; ello, con el propósito de reconocer la relevancia de los individuos en las compañías, dentro de los diversos sectores económicos.

\section{Fundamentación teórica}

A continuación, se relacionarán de manera sucinta las categorías de análisis que se consideran pertinentes para el desarrollo de la investigación propuesta.

Generaciones sociales. Se entiende como generación al grupo de personas nacidas en un mismo periodo de tiempo, quienes comparten hechos históricos y sociales similares; sucesos que a su vez señalan un antes y un después en la vida de cada grupo generacional. Estos eventos significativos pueden ser guerras, crisis económicas, caída de paradigmas, innovaciones tecnológicas o cambios climáticos, sucesos que a su vez influyen en las actitudes, los comportamientos y los conocimientos de las personas. En relación con lo anterior, actualmente se presenta un desafío en las organizaciones debido a la diversidad de generaciones que aportan sus puntos de vista; dichas diferencias pueden influir en aspectos como la comunicación dentro de la organización(Díaz et al., 2017).

A través del tiempo se han identificado distintos grupos generacionales, los cuales han sido clasificados por diferentes autores de acuerdo con intervalos de años(Hernández, 2014). Para este estudio, la población objetivo se ha centrado en las personas clasificadas entre las generaciones $X$ y Y, es decir, los nacidos entre los años 1965 y 1994, quienes tienen, a la fecha de esta investigación, entre 26 y 55 años.

- Generación X. Esta generación comprende a los individuos que ocupan en la actualidad cargos de mando medio y alto dentro de las organizaciones. Este grupo generacional percibe el trabajo de manera diferente a como lo percibieron sus padres, los baby boomers, en tanto conciben el trabajo como la forma de alcanzar sus propósitos personales, mientras tratan de tener un equilibrio entre su vida personal y su vida laboral. Una de las características de esta generación es la gran aceptación a la diversidad y al cambio, lo cual genera beneficios para las organizaciones; además de lo anterior, dicha generación posee lealtad corporativa, valora las relaciones con sus compañeros, aprecia el reconocimiento de sus jefes y se enfoca en exponer sus motivaciones y proyecciones en el trabajo(Díaz et al., 2017).

- Generación Y. En esta generación se ubican las personas que, principalmente, tienen pensamientos relacionados con el ideal de cambiar el mundo; buscan ser más honestos, más ecológicos y más exitosos. Una de las cosas más destacadas de esta generación es su afinidad por el mundo digital, motivo por el cual esta se considera como la primera generación, dentro del mercado laboral, que cuenta con un conocimiento de las herramientas tecnológicas mayor que el de sus superiores. Dicha generación es considerada como parte esencial en la fuerza laboral; muchos de ellos se encuentran establecidos o incursionando en cargos de 
liderazgo; in embargo, constantemente consideran buscar nuevas oportunidades; por otra parte, no se siente a gusto con las estructuras rígidas y requieren cargos variados e interesantes en el trabajo, los cuales les permitan obtener crecimiento personal y profesional (Díaz etal., 2017).

- Capital intelectual. Sánchez afirma que "el capital intelectual es la suma de todos los conocimientos que pueden ser convertidos en beneficios en el futuro, el mismo está formado por ideas, las tecnologías, los programas informáticos, los procesos y los diseños" (2020, p.15). Se entiende también como capital intelectual al activo intangible e intransferible que tiene la capacidad de producir valor a la organización, convirtiendo los conocimientos en una ventaja competitiva; así mismo, interactúa tanto con los activos naturales, como en las relaciones de la empresa con los agentes externos, procedimientos y políticas internas que, en conjunto, generan ventajas sostenibles y sustentables a través del tiempo (Villegas et al., 2017).

A continuación, se explican las tres dimensiones a saber, el capital humano, el capital relacional y el capital estructural, las cuales a su vez constituyen la variable del capital intelectual.

- Capital humano. Es el conjunto de capacidades, de conocimientos y de valores, que propenden a la innovación, teniendo presente la formación académica y las prácticas de los empleados(Archibold \& Escobar, 2015). Existen aspectos específicos para medir la dimensión del capital intelectual; algunos de ellos son la actitud y la aptitud. La primera es conocida como el comportamiento positivo o negativo de un individuo al realizar una actividad, es decir, la manera de actuar o el carácter ante cualquier situación. Así mismo, la aptitud del trabajador se define como el grupo de habilidades o atributos mentales y físicos, que generan acciones sobresalientes; en otras palabras, la actitud es la manera de hacer y la aptitud la capacidad para hacer(Sánchez, 2020).

- Capital relacional. Es el valor agregado por las capacidades de la organización, que originan beneficios de carácter financiero, administrativo y competitivo, por medio de sus relaciones con las diversas partes interesadas(Archibold \& Escobar, 2015). Esta dimensión refiere a todas las relaciones que se generan dentro de una organización. Edvinsson Leif \& Malone (1999) "consideran que esta dimensión está integrada, principalmente, por el valor generado por las relaciones de la empresa con sus clientes" (Sánchez Medina et al., 2007). Por su parte, Brooking (1997) "denomina a esta dimensión activos de mercado y la define como aquellos recursos que otorgan a la empresa ventaja competitiva en virtud del potencial que se deriva de los bienes inmateriales que guardan relación con el mercado" y, a modo de aclaración precisa, "se debe reflejar que en valor de las relaciones de la empresa con sus clientes influyen los miembros de la organización que establecen y mantienen dichas relaciones, por tanto, en su gestión siempre habrá un factor de incertidumbre mayor la que tienen los activos pertenecientes al capital estructural" (Sánchez Medina et al., 2007). 
- Capital estructural. Este tipo de capital establece las capacidades de la empresa con relacióna la productividad y a la competitividad ocasionada por la cultura organizacional; así mismo, el capital estructural hace referencia al desarrollo tecnológico facilitador de las operaciones diarias, al mejoramiento de los procesos y al posicionamiento en el mercado, lo que determina las habilidades organizacionales que generan conocimientos, en función de obtener beneficios de tipo económico(Archibold \& Escobar, 2015).

\section{Prueba U de Mann-Whitney}

La prueba no paramétrica $U$ de Mann-Whitney suele utilizarse para verificar la similitud y la diferencia entre dos muestras (Jiménez, Landeros, \& Huerta, 2015). Esta prueba se basa en diferencias de rango y es equivalente al parámetro prueba t de Student, que se utiliza para variables cuantitativas con distribución normal(Romero Saldaña, 2013).

\section{Metodología}

Tipo de estudio. El presente trabajo tiene un enfoque cuantitativo, en tanto se considera la aplicación de tratamientos estadísticos a los datos recopilados por el instrumento de recolección de información (Gómez, Sánchez y Torres, 2016). La investigación es no experimental, porque las variables de investigación no se han modificado, solo se seleccionan y se observan (Duran, 2018). Por consiguiente, en el actual estudio no se tiene un experimento. Adicionalmente es de corte transversal, ya que los datos se recolectan en un solo momento 0 en un tiempo único (Cabrera et al., 2014). Por lo anterior, la investigación aplica una sola vez los instrumentos a la población de estudio, permitiendo evaluar los niveles de capital intelectual entre las poblaciones objeto de estudio.

Participantes. La encuesta Nuevo Marco 2005 proyectó que para el trimestre que va de julio a septiembre del año 2019, Bucaramanga y su área metropolitana contaría con 1'097.068 de ciudadanos, de los cuales, 563.867 pertenecerían a la población ocupada del país, siendo 391.088, las personas pertenecientes a las generaciones X y Y, es decir, individuos que estarían entre los 26 y 55 años de edad (DANE, 2005). Para esta investigación se realizó un muestreo aleatorio simple probabilístico. La población total encuestada fue de 248 trabajadores, pertenecientes a las zonas geográficas objeto de estudio.

Procedimiento. Para realizar el presente estudio, en primer lugar se realiza de manera aleatoria la medición del nivel de capital intelectual de 248 habitantes, correspondientes a las generaciones $X$ y Y, quienes trabajan en la ciudad de Bucaramanga y en su área metropolitana; para lo anterior, se utiliza el cuestionario de capital intelectual basado en el modelo intelect. A continuación, con el objetivo de analizar su comportamiento dentro de las organizaciones, se estudian las dimensiones propias de la variable estudio, a través de un análisis descriptivo de los datos para lo cual, se emplea la prueba U de Mann-Whitney, con el propósito de identificar las semejanzas y las diferencias entre los colaboradores evaluados, pertenecientes a las generaciones X y $Y$, frente $a$ la variable de capital intelectual. Lo anterior, con el fin de brindar a las organizaciones de los 
municipios objeto de estudio, un insumo que les permita conocer las diferencias generacionales entre sus trabajadores y, a partir de ello, puedan realizar una mejor gestión del talento humano, lo que a su vez les permite lograr un crecimiento organizacional y un avance económico en las empresas.

Materiales e instrumentos. El instrumento de recolección de la información aplicado fue el propuesto por Alama (2008), a partir del cual se reconocen elementos favorables en el alfa de Cronbach en las dimensiones, puesto que el rango oscila entre 0,75 y 0,90, siendo su validez mejorada por medio de pruebas estadísticas de análisis factorial confirmatorio; así mismo, es pertinente referir que el modelo orientador es el intelec, el cual fue un sistema realizado por un equipo de trabajo de Euroforum en 1998, bajo la premisa de lograr una cohesión entre la estrategia de la empresa y los activos intangibles (Martínez Moreno, 2017).

\section{Resultados}

El estudio del capital intelectual en los colaboradores de la generación X y Y se fundamentó en las dimensiones que componen la variable objeto de estudio, entendidas estas como el capital humano, el capital estructural y el capital relacional; a su vez, se contemplaron como unidades de análisis la muestra general y la particular, el género, el nivel educativo y el municipio donde se ejerce una determinada labor.

Los hallazgos encontrados en relación a la totalidad de la población objeto de estudio (Figura1), es decir, la correspondiente a los 248 colaboradores de la generación X y Y que fueron encuestados, en correspondencia con cada una de las dimensiones consideradas para el análisis de la variable "capital intelectual". Como se puede observar, el factor capital estructural obtuvo una ponderación de 4,31; el capital humano obtuvo una ponderación de 4,29 y el capital relacional una ponderación de 4,25. Por lo tanto se obtiene como resultado que el factor que alcanzó el mayor nivel fue el referente a la dimensión de capital estructural, lo que permite inferir una marcada tendencia a la productividad y a la competitividad en las unidades empresarial; ello, producto de la continua necesidad de lograr mejores resultados en mercados cada vez más competitivos.

Figura 1.

Capital intelectual de las Generaciones Xy Y. Fuente: elaboración propia.

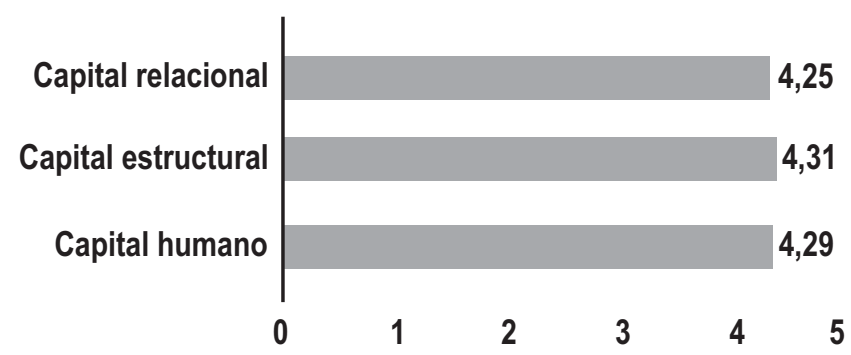

La unidad de análisis generación Xabarca una muestra total de 107 colaboradores; esto quiere decir 
que el $43 \%$ de las personas encuestadas tenían entre 40 y 55 años. La Figura 2 muestra los resultados para cada dimensión considerada para el análisis de la variable "capital intelectual". La dimensión "capital humano" obtuvo una ponderación de 4,358; "capital estructural" de 4,353 y "capital relacional" de 4,30, lo cual evidencia que los colaboradores de la generación X poseen mejores características, en relación con la variable "capital intelectual", ya que cumplen con las actividades que le son recomendadas por la organización, y además se destacan por su amplio conocimiento en el área de trabajo.

Figura 2.

Niveles de capital intelectual de la generación X.

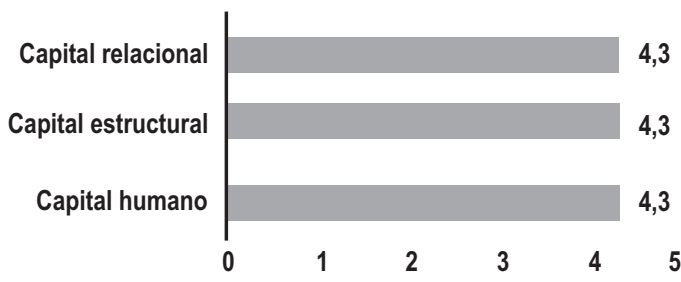

Fuente: elaboración propia.
Figura 3.

Niveles de capital intelectual de la generación Y.

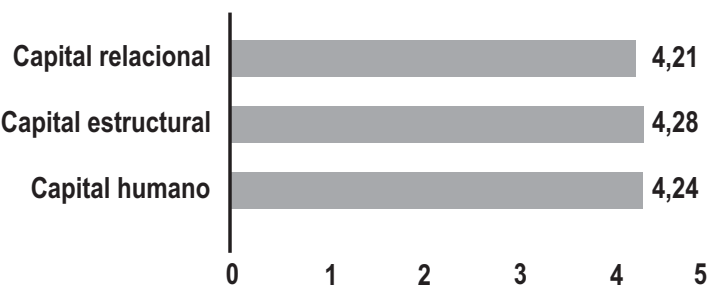

Fuente: elaboración propia.

Por otra parte, la unidad de análisis generación Y, contó con una muestra total de 141 trabajadores; esto quiere decir que el $53 \%$ de los colaboradores encuestados tenía entre 26 y 39 años. La Figura 3 muestra el resultado de cada una de las dimensiones que se tuvo en cuenta para el análisis de la variable "capital intelectual" en esta generación; así entonces, como se muestra, la dimensión "capital humano" obtuvo una ponderación de 4,24; "capital estructural" de 4,28 y "capital relacional" de 4,21, lo cual evidencia también que los colaboradores poseen características favorables en el entorno laboral, ello, debido en gran medida a que las organizaciones tienen marcos estratégicos sólidos, los cuales son ampliamente difundidos entre la totalidad de los interesados.

\section{Niveles de capital intelectual por unidad de análisis}

A continuación se muestran los niveles de capital intelectual de las generaciones $X$ y $Y$, los cuales son presentados teniendo en cuenta el género como unidad de análisis. El número de hombres encuestados que pertenecen a las generaciones $X$ y $Y$ es de 57 y 79 colaboradores respectivamente, para una muestra total de 136 hombres, lo cual representa el $55 \%$ del total de los colaboradores encuestados. Así mismo, el número de mujeres encuestadas para este estudio, las cuales pertenecen a las generaciones $X$ y $Y$, es de 50 y 62 trabajadoras respectivamente, para una muestra total de 112 mujeres, lo cual representa al 45\% del total de los colaboradores encuestados.

La Tabla 1 muestra los resultados de las dimensiones estimadas para el análisis de la variable "capital intelectual", de acuerdo con el género y con la generación social. Como se aprecia, los hombres y las mujeres de la generación $\mathrm{X}$ poseen características superiores relacionadas con el 
Tabla 1.

Nivel de capital intelectual por género.

\begin{tabular}{lccccc}
\hline Unidad de análisis & \multicolumn{3}{c}{ Género masculino } & \multicolumn{3}{c}{ Género femenino } \\
\hline \multicolumn{1}{c}{ Generación } & $\mathrm{X}$ & $\mathrm{Y}$ & $\mathrm{X}$ & $\mathrm{Y}$ \\
\hline Dimensiones & \multicolumn{5}{c}{ Puntaje } \\
\hline Capital relacional & 4,32 & 4,23 & 4,29 & 4,19 \\
Capital estructural & 4,37 & 4,24 & 4,33 & 4,32 \\
Capital humano & 4,35 & 4,22 & 4,36 & 4,27 \\
\hline
\end{tabular}

Fuente: elaboración propia.

"capital intelectual", las cuales son ligeramente mayores a las de los sujetos que pertenecen a la generación $Y$. Para la generación $X$, las dimensiones con puntuaciones superiores en relación con el "capital intelectual" son las de "capital estructural" y "capital humano", con ponderaciones de 4,37 y 4,36 en hombres y en mujeres respectivamente. Sin embargo, para el caso de la generación Y, la dimensión que refleja la mayor ponderación es la referente al "capital estructural", con puntuaciones de 4,24 y 4,32 en hombre y mujeres respectivamente.

Por otra parte, en la Tabla 2 se presentan los niveles de capital intelectual de las generaciones X y Y, de acuerdo con el nivel educativo de los colaboradores encuestados, variable que se tiene en cuenta como unidad de análisis. Del mismo modo, esta tabla muestra los resultados de cada una de las dimensiones que se tuvo en cuenta para el análisis de la variable "capital intelectual". Como se observa, los colaboradores de la generación $X$ que cuentan con estudios de posgrado poseen calificaciones superiores en relación con el "capital intelectual", respecto de los de la generación Y; lo anterior con puntuaciones de "capital relacional", "capital estructural" y "capital humano" de 4,51, 4,54 y 4,55, respectivamente. Mientras tanto, se encuentra que entre los sujetos pertenecientes a la generación $\mathrm{X}$, los colaboradores con más bajas puntuaciones son los que cuentan con estudios técnicos profesionales o tecnológicos, quienes obtuvieron puntuaciones de "capital relacional", "capital estructural" y "capital humano" de 4,01, 4,17 y 4,14 respectivamente. Así mismo, de acuerdo con los resultados presentados en dicha tabla, se puede evidenciar que la dimensión de "capital

Tabla 2.

Nivel de capital intelectual de acuerdo con el nivel educativo.

\begin{tabular}{|c|c|c|c|c|c|c|c|c|}
\hline \multirow{2}{*}{$\begin{array}{l}\text { Unidad de análisis } \\
\text { Generación }\end{array}$} & \multicolumn{2}{|c|}{ Educación media } & \multicolumn{2}{|c|}{$\begin{array}{c}\text { Educación técnica } \\
\text { o tecnológica }\end{array}$} & \multicolumn{2}{|c|}{$\begin{array}{c}\text { Educación } \\
\text { universitaria }\end{array}$} & \multicolumn{2}{|c|}{ Postgrado } \\
\hline & $X$ & $Y$ & $x$ & $Y$ & $X$ & $Y$ & $X$ & $Y$ \\
\hline Dimensiones & \multicolumn{8}{|c|}{ Puntaje } \\
\hline Capital relacional & 4,3 & 4,19 & 4 & 4,25 & 4,3 & 4,21 & 4,5 & 4,11 \\
\hline Capital estructural & 4,3 & 4,27 & 4,2 & 4,35 & 4,4 & 4,25 & 4,5 & 4,26 \\
\hline Capital humano & 4,3 & 4,25 & 4,1 & 4,3 & 4,4 & 4,17 & 4,6 & 4,23 \\
\hline
\end{tabular}

Fuente: elaboración propia. 
intelectual" con mayor nivel de ponderación por cada unidad de análisis es la referente al "capital estructural", independientemente del nivel educativo y de la generación a la que pertenezcan los colaboradores.

La tabla 3 presenta los niveles de "capital intelectual" de las generaciones X y Y de acuerdo con el lugar donde laboran los colaboradores variable que se presenta como unidad de análisis. Esta tabla muestra los resultados de cada una de las dimensiones que se tiene en cuenta para el análisis de la variable "capital intelectual". Como se observa, los colaboradores que laboran en el municipio de Piedecuesta y que pertenecen a las generaciones $X$ y $Y$, poseen en promedio puntaciones superiores de "capital intelectual", respecto de los que laboran en los municipios de Girón y Floridablanca. Por su parte, los trabajadores pertenecientes a la generación $\mathrm{X}$ que laboran en el municipio de Bucaramanga, presentan niveles de ponderación similares a los de quienes laboran en el municipio de Piedecuesta, y tienen niveles ligeramente mayores a los de la generación Y, que laboran en el municipio de Bucaramanga. Por otra parte, los trabajadores pertenecientes a las generaciones $X$ y $Y$ que laboran en el municipio de Girón son quienes, en promedio, poseen calificaciones inferiores, en relación con el "capital intelectual", ello respecto de los que trabajan en los demás municipios. Así mismo, de acuerdo con los resultados encontrados, se puede evidenciar que la dimensión de "capital intelectual" con mayor nivel de ponderación en general es la referente a "capital humano", independientemente del municipio donde laboren los empleados y de la generación a la que pertenezcan los colaboradores. Por tal motivo, se reconoce una línea potencial de investigación complementaria, dentro del marco de identificación de los elementos causales de tal comportamiento, específicamente entorno al municipio de Piedecuesta.

\section{Prueba U de Mann-Withney}

Finalmente se aplicó la prueba de $U$ de Mann-Withney, con el fin de reconocer la homogeneidad o la heterogeneidad entre las generaciones $X$ y $Y$, frente a cada una de las dimensiones que componen la variable objeto de estudio: "capital intelectual" (Kiss \& Gastelú, 2015). Los resultados obtenidos en la Tabla 4 permiten identificar que las dimensiones de "capital humano", es decir, "capital estructural" y "capital relacional", se presentan de la misma manera en los trabajadores de ambas generaciones; por consiguiente, se puede generar, en una instancia posterior, recomendaciones y estrategias con

Tabla 3.

Nivel de capital intelectual de acuerdo con el lugardonde se labora.

\begin{tabular}{cccccccccc}
\hline Unidad de análisis & \multicolumn{3}{c}{ Bucaramanga } & \multicolumn{3}{c}{ Floridablanca } & \multicolumn{2}{c}{ Girón } & \multicolumn{2}{c}{ Piedecuesta } \\
\hline Generación & $\mathrm{X}$ & $\mathrm{Y}$ & $\mathrm{X}$ & $\mathrm{Y}$ & $\mathrm{X}$ & $\mathrm{Y}$ & $\mathrm{X}$ & $\mathrm{Y}$ \\
\hline Dimensiones & & \multicolumn{7}{c}{ Puntaje } \\
\hline Capital relacional & 4,40 & 4,2 & 4,28 & 4,1 & 4,21 & 4,20 & 4,30 & 4,47 \\
Capital estructural & 4,47 & 4,2 & 4,25 & 4,3 & 4,2 & 4,2 & 4,4 & 4,51 \\
Capital humano & 4,49 & 4,2 & 4,29 & 4,3 & 4,24 & 4,1 & 4,4 & 4,42 \\
\hline
\end{tabular}

Fuente: elaboración propia. 
Tabla 4.

Resultados de la prueba U de Mann-Withney.

\begin{tabular}{lccc}
\hline \multicolumn{1}{c}{ Dimensión } & Capital humano & Capital estructural & Capital relacional \\
\hline U de Mann-Whitney & 6948,5 & 7030,5 & 6966,5 \\
W de Wilcoxon & 16959,5 & 17041,5 & 16977,5 \\
Z & $-1,065$ & $-0,918$ & $-1,033$ \\
Sig. asintótica (bilateral) & 0,287 & 0,358 & 0,301 \\
\hline
\end{tabular}

Fuente: elaboración propia.

el fin de fomentar dicha práctica, dentro del personal de las compañías de los diversos sectores económicos del municipio de Bucaramanga y de su área metropolitana.

\section{Discusión}

Teniendo en cuenta el total de colaboradores encuestados en Bucaramanga y en su área metropolitana, los resultados de la variable "capital intelectual" que se encuentra en las generaciones $X$ y $Y$, permiten identificar que los trabajadores poseen altos niveles de capital intelectual; esto debido quizá a que en gran medida las organizaciones poseen visión y valores, y a que además fomentan la formación individual de su capital humano. Así mismo, la dimensión "capital estructural" obtuvo la ponderación más alta, lo que permite inferir que los trabajadores son tenidos en cuenta en diversos procesos de toma de decisión, por lo que pueden demostrar su alto nivel de conocimiento. Así mismo, se observa que el factor "capital relacional" presenta la menor ponderación, lo cual permite deducir que los colaboradores no se enfocan en la relación con los clientes, lo cual produce que dentro del mercado regional, las organizaciones no se encuentren ampliamente posicionadas. En tal sentido, las mismas poseen poca intención hacia el cooperativismo, ya que no generan alianzas estratégicas. Lo anterior a su vez implica que los trabajadores no poseen los conocimientos necesarios para que las empresas donde laboran, trabajen en conjunto con otras.

Ahora bien, particularmente analizando los resultados presentados por cada una de las generaciones que fueron analizadas, se puede deducir, en términos generales, que el nivel de "capital intelectual" que muestran los trabajadores de la generación X es levemente superior al de los trabajadores de la generación Y; no obstante, este no es totalmente significativo; en tal sentido, se entiende que la dimensión con superiores brechas es la de "capital humano", lo que permite inferir que son los colaboradores pertenecientes a esta generación, los que poseen en su generalidad un mayor sentido de pertenencia con la organización, ya que recomiendan los productos de las compañías donde laboran. 


\section{Conclusiones}

El "capital intelectual" es el mecanismo de conocimiento y de motivación que crea valor dentro de una organización. Este influye en la competitividad de las empresas y mejora su posicionamiento frente a la competencia, lo cual le permite a las mismas alcanzar mayores utilidades, optimizar la capacidad de la planta y lograr una menor rotación de los colaboradores. Por lo anterior, es de vital importancia conocer las habilidades de los colaboradores que pertenecen a cada una de las generaciones, con el fin de optimizar la gestión del talento humano, dentro de las organizaciones.

Por su parte, el nivel de capital intelectual presente en las generaciones objeto de estudio, evidencia que los colaboradores poseen un sobresaliente capital intelectual, debido, en gran medida, a que se infiere que las organizaciones que poseen visión y valores, fomentan el aprendizaje continuo de los colaboradores (Vega Martínez et al., 2019). Así mismo, en las organizaciones de Bucaramanga y de su área metropolitana, se observa que los trabajadores son tenidos en cuenta en diversos procesos de toma de decisión, lo que demuestra su alto nivel de conocimiento, resaltando que el capital estructural de las organizaciones, fomenta individuos con alto nivel de capital intelectual. Por otro parte, se observó que la dimensión de capital relacional es la más débil en las organizaciones, dado que los individuos poseen poca intención hacia el cooperativismo, lo cual en última instancia afecta las relaciones externas de las empresas. Así mismo, los resultados presentados a partir de la prueba U- Mann Whitney permitieron determinar que las tres dimensiones del capital intelectual, esto es, capital humano, capital estructural y capital relacional, mostraron resultados semejantes para ambas generaciones, por lo que se entiende que los mismos no dependen de la generación a la que pertenezca el colaborador, lo cual lleva a concluir que no existen diferencias entre dichas generaciones, frente a la variable de capital intelectual.

Finalmente, se entiende que las futuras líneas de trabajo, en el constructo objeto de estudio, precisan la formulación de propuestas de mejora del capital intelectual a nivel organizacional, considerando los elementos diferenciables de las diversas unidades empresariales.

\section{Referencias bibliográficas}

Allama, E. (2008). Capital intelectual y resultados empresariales en las empresas de servicios profesionales de España. [Tesis].

Archibold, W., \& Escobar, A. (2015). Capital intelectual y gestión del conocimiento en las contralorías territoriales del departamento del Atlántico. Dimensión Empresarial, 13(1), 133-146.

Azofra, V., Ochoa, M. L., Prieto, B., \& Santidrián, A.(2017). Creando valor mediante la aplicación de modelos de capital intelectual. Innovar: Revista de Ciencias Administrativas y Sociales, 27(65), 25-38.

Bermúdez, S., \& Hernández, A. (2019). Diagnosis of intellectual capital in a Mexican textile manufacturing company. Revista EAN, 86, 57-76. 
Brooking, A. (1997). El capital intelectual: el principal activo de las empresas del tercer milenio. Paidós.

Cabrera, L., Bethencourt, J. T., González, M., \& Álvarez, P. (2014). Un estudio transversal retrospectivo sobre prolongación y abandono de estudios universitarios. RELIEVE-Revista Electrónica de Investigación y Evaluación Educativa, 12(1).

DANE. (2005). Encuesta nuevo marco 2005 - julio a septiembre de 2019.

Díaz, C., López, M., \& Roncallo, L. (2017). Entendiendo las generaciones: una revisión del concepto, clasificación y características distintivas de los baby boomers, $X$ y millennials. Clío América, 11(22), 188-204. https://doi.org/https://doi.org/10.21676/23897848.2440

Durán Velásquez, L. (2018). Estrategia de gestión basada en el cuadro de mando integral para la empresa Herrametal C.A. ubicada en municipio Iribarren, Estado Lara. Enfoque Disciplinario, 3(1), 49-71. http://enfoquedisciplinario.org/revista/index.php/enfoque/article/view/12

García-Méndez, S., Galvis-Rojas, Y. P., \& Ortiz-Olago, J. A. (2020). Relación entre clima organizacional y riesgo psicosocial intralaboral de una empresa de transporte en Bucaramanga. I+D. Revista de Investigaciones, 15,(1), 61-68. https://doi.org/10.33304/revinv.v15n1-2020007

Gómez-Cano, C., Aristizabal-Valbuena, C., \& Fuentes-Gómez, D. (2017). Importancia de la información financiera para el ejercicio de la gerencia. Desarrollo Gerencial, 9(2), 88-101. https://doi.org/10.17081/dege.9.2.297

Gómez, C., Sánchez, V., Torres, L. (2016). Análisis del impacto del Programa Jóvenes en Acción de la Universidad de laAmazonia período 2014-2015. Revista FACCEA, 6(1), 58-69.

Guelmes, E. L., \& Nieto, L. E. (2015). Algunas reflexiones sobre el enfoque mixto de la investigación pedagógica en el contexto cubano. Revista Universidad y Sociedad, 7(1), 23-29.

Hernández Barandica, L. (2014). La generación y (nativos tecnológicos) y su encaje en las organizaciones: aproximación teórico-práctica. Universidad Pontificia Comillas ICAI-ICADE.

Jiménez-Ochoa, S., Landeros-Pérez, M. E., \& Huerta-Franco, M. (2015). Efecto del masaje terapéutico como cuidado de enfermería en la capacidad funcional del adulto mayor. Enfermería Universitaria, 12(2), 49-55.

Leif, Edvinsson y Malone, M. S. (1999). El Capital Intelectual: Cómo Identificar y Calcular el Valor de Los Recursos. Ediciones Gestión 2000.

Limache, E. M. (2017). Capital Intelectual en la Competitividad de las MIPYMES de la Región Tacna2015. Universidad Privada de Tacna.

Martínez Moreno, L. (2017). Medición y gestión del capital intelectual en las instituciones de educación 
superior (IES) de las fuerzas militares de Colombia. (Tesis doctoral. Universidad Autónoma de Madrid, Facultad de Ciencias Económicas y Empresariales). https://dialnet.unirioja.es/servlet/te sis?codigo $=147066$.

Oblinger, D., Oblinger, J. L., \& Lippincott, J. K. (2005). Educating the net generation (Vol. 1). Boulder, Colo.: EDUCAUSE, c2005.

Romero Saldaña, M. (2013). Contraste de Hipótesis Comparación de dos medias independientes mediante pruebas no paramétricas: Prueba U de Mann-Whitney. Revista Enfermería Del Trabajo, $3(2), 77-84$.

Sánchez, L. (2020). El capital intelectual y la productividad en las empresas del sector carrocero de la provincia de Tungurahua.

Sánchez-Medina, A. J., Melián-González, A., y Hormiga-Pérez, E. (2007). El concepto de capital intelectual y sus dimensiones. Investigaciones europeas de dirección y economía de la empresa, 13(2), 97-111

Sarkis, B. (2017). La diversidad organizacional. Revista de La Federación Colombiana de Gestión Humana-Acrip Nacional, 105, 15-20.

Vega Martínez, J. E., Martinez Serna, M. del C., \& Párga Montoya, N. (2019). Influencia del aprendizaje organizacional y los resultados de las Pymes. Investigación Administrativa, 48, 1-18. https://doi.org/10.35426/iav48n124.06.

Villegas, E., Hernández, M. A., \& Salazar, B. C. (2017). La medición del capital intelectual y su impacto en el rendimiento financiero en empresas del sector industrial en México. Contaduría y Administración, 62(1), 184-206 